\title{
SOME CHARACTERIZATIONS OF TOTIENTS
}

\author{
PENTTI HAUKKANEN \\ Department of Mathematical Sciences \\ University of Tampere, \\ P.O. Box 607, FIN-33101 Tampere, FINLAND
}

(Received October 4, 1994 and in revised form January 27, 1995)

\begin{abstract}
An arithmetical function is said to be a totient if it is the Dirichlet convolution between a completely multiplicative function and the inverse of a completely multiplicative function. Euler's phi-function is a famous example of a totient. All completely multiplicative functions are also totients. There is a large number of characterizations of completely multiplicative functions in the literature, while characterizations of totients have not been widely studied in the literature. In this paper we nresent several arithmetical identities serving as characterizations of totients. We also introduce a new concrete example of a totient.
\end{abstract}

KEY WORDS AND PHRASES. Multifiicative arithmetical functions, totient functions, Euler's phi-function, Dirichlet convolution, arithmetical identities

1992 AMS SUBJETT CLASSIFICATION CODE. 11A25

\section{INTRODUCTIN.}

The Dirichlet convolution of arithmetical functions $f$ and $g$ is defined by

$$
(f * g)(n)=\sum_{d \mid n} f(d) g(n / d)
$$

If $f(1) \neq 0$, then the Dirichlet inverse of $f$ is the arithmetical function $f^{-1}$ satisfying

$$
f * f^{-1}=f^{-1} * f=e_{0},
$$

where $e_{0}(1)=1$ and $e_{0}(r)=0$ for $n>1$. An arithmetical function is said to be multiplicative if $f(1)=1$ and $f(m n)=f(m) f(n)$ whenever $(m, n)=1$. A multiplicative function $f$ is totally determined by its values $f\left(p^{e}\right)$ at all prime powers $p^{e}$. A multiplicative function $f$ is also totally determined by its generating series

$$
f_{p}(x)=\sum_{n=0}^{\infty} f\left(p^{n}\right) x^{n}
$$

at all primes $p$. A multiplicative function $f$ is said to be completely multiplicative if $f(m n)=$ $f(m) f(n)$ for all $m$ and $n$. A completely multiplicative function $f$ is totally determined by its values $f(p)$ at all primes $p$.

In this paper we cunsider multiplicative functions $f$ which can be written in the form

$$
f=f_{t} * f_{v}^{-1}
$$


where $f_{t}$ and $f_{v}$ are completely multiplicative functions. Such functions $f$ are called totients ([14], p. 612). The functions $f_{t}$ and $f_{v}$ are said to be integral and inverse components of $f$, respectively. Note that $f_{t}$ and $f_{v}$ are not always unique. Namely, if there exists a prime $p$ such that $f\left(p^{e}\right)=0$ for all positive integers $e$, then it suffices that $f_{t}(p)$ and $f_{v}(p)$ satisfy $f_{t}(p)=f_{v}(p)$. Otherwise $f_{t}(p)$ and $f_{v}(p)$ are uniquely determined.

Totients belong to the class of rational arithmetical functions. Namely, Vaidyanathaswamy [14] defined an arithmetical function $f$ to be a rational arithmetical function of degree $(r, s)$ if there exist completely multiplicative functions $g_{1}, g_{2}, \ldots, g_{r}$ and $h_{1}, h_{2}, \ldots, h_{s}$ such that

$$
f=g_{1} * g_{2} * \cdots * g_{r} * h_{1}^{-1} * h_{2}^{-1} * \cdots * h_{s}^{-1}
$$

Totients are thus rational arithmetical functions of degree $(1,1)$, and completely multiplicative functions are rational arithmetical functions of degree $(1,0)$. Note that all completely multiplicative functions are totients with $f_{v}=e_{0}$.

There is a large number of characterizations of completely multiplicative functions in the literature. For example, each of the following four conditions is a necessary and sufficient condition for a multiplicative function to be completely multiplicative.

(i) $f^{-1}\left(p^{e}\right)=0$ for all primes $p$ and integers $e \geq 2$,

(ii) $f\left(p^{e}\right)=f(p)^{e}$ for all primes $p$ and integers $e \geq 1$,

(iii) $f^{-1}=\mu f$, where $\mu$ is the Möbius function,

(iv) $f_{p}(x)=\frac{1}{1-f(p) x}$ for all primes $p$.

For further characterizations of completely multiplicative functions, see e.g. [1].

Characterizations of totients have not much been studied in the literature. Wall and Hsu [15] have given a characterization. Namely, a multiplicative function $f$ is a totient if and only if $f(p), f\left(p^{2}\right), f\left(p^{3}\right), \ldots$ is a geometric progression for each prime $p$. The purpose of this paper is to present some further characterizations. Most of our characterizations have been developed from known properties of some concrete examples of totients.

Euler's function $\phi(n)$ is a famous concrete totient, which is defined as the number of integers $x(\bmod n)$ such that $(x, n)=1$. It is well-known that $\phi=N * \mu$, where $N(n)=n$ for all $n$. Thus $\phi$ is a totient with $\phi_{t}=N$ and $\phi_{v}=e$, where $e \equiv 1$. There is a large number of generalizations and analogues of Euler's totient in the literature. Many of the generalizations and analogues are defined combinatorially. For example, the Jordan totient $J_{k}(n)$ is defined as the number of ordered $k$-tuples $\left\langle x_{1}, \ldots, x_{k}\right\rangle(\bmod n)$ such that $\operatorname{gcd}\left(x_{1}, x_{2}, \ldots, x_{k}, n\right)=1$. Many of the generalizations and analogues also possess the structure of a totient in the sense of Vaidyanathaswamy [14]. For example, the Jordan totient can be written as $J_{k}=N^{k} * \mu$, where $N^{k}(n)=n^{k}$ for all $n$. For further totients reference is made to the books by McCarthy [9] and Sivaramakrishnan [11] and to the paper by Sivaramakrishnan [12]. In Section 3 of this paper we introduce a new example of a totient denoted by $\theta_{(k)}$. The characterizations of Section 2 can be used in deciding whether a function is a totient and to obtain identities for concrete totients. We apply the results of Section 2 to the function $\theta_{(k)}$.

\section{CHARACTERIZATIONS}

In this section we present necessary and sufficient conditions for an arithmetical function $f$ to be a totient. These conditions may not be considered entirely new, since they are either conceived from properties of some concrete examples $\epsilon$ totients or known to be necessary conditions for totients. For brevity, we assume throughout this section that $f(1)=1$. 
THEOREM 1. An arithmetical function $f$ is a totient if, and only if, $f$ is multiplicative and for each prime $p$ there exists a complex number $a(p)$ such that

$$
f\left(p^{e}\right)=f(p)(a(p))^{e-1} \text { for all } e \geq 1 .
$$

In this case $f_{t}(p)=a(p)$.

PROOF. Theorem 1 follows after some manipulation by definition of totients and properties of completely multiplicative functions. We omit the details.

COROLLARY 1. If $f$ and $g$ are totients, then $f g$ is a totient with $(f g)_{t}=f_{t} g_{t}$.

COROLLARY 2 ([15]). A multiplicative function $f$ is a totient if, and only if, for each prime $p, f(p), f\left(p^{2}\right), f\left(p^{3}\right), \ldots$ is a geometric progression.

REMARK. Several further characterizations could be derived from Theorem 1 using the formula $f(p)=f_{t}(p)-f_{v}(p)$ for all primes $p$.

THEOREM 2. An arithmetical function $f$ is a totient if, and only if, there exists completely multiplicative functions $g$ and $h$ such that

$$
f(n)=\sum_{x(\bmod n)} \sum_{d \mid(x, n)} g(n / d) h^{-1}(d)(d / n) .
$$

In this case $f_{t}=g$ and $f_{v}=h$.

REMARK. For the Euler totient $\phi$, equation (2) reduces to

$$
\phi(n)=\sum_{x(\bmod n)} e_{0}((x, n)),
$$

which, in fact, is the definition of $\phi$.

PROOF. If $f$ is a totient, then

$$
f(n)=\left(f_{t} * f_{v}^{-1}\right)(n)=\sum_{d \mid n} f_{t}(n / d) f_{v}^{-1}(d)(d / n) \sum_{e=1}^{n / d} 1=\sum_{x=1}^{n} \sum_{d \mid(x, n)} f_{t}(n / d) f_{v}^{-1}(d)(d / n) .
$$

We thus arrive at (2). The converse part follows similarly.

THEOREM 3. An arithmetical function $f$ is a totient if, and only if, there exists a multiplicative function $h$ such that

$$
f(m) f(n)=\sum_{d \mid(m, n)} f(m n / d) h(d) \mu(d) .
$$

In this case $f_{v}=h$.

REMARK. Sivaramakrishnan ([10], p. 27) and Sugunamma ([13], p. 40) have noted that (3) is a necessary condition for totients.

PROOF. Formula (3) can be proved by showing that both sides of (3) are multiplicative functions in two variables and by studying prime powers. Conversely, taking $(m, n)=1$ in (3) shows that $f$ is multiplicative. Taking $m=p^{e-1}, n=p(e \geq 2)$ in (3) gives

$$
f\left(p^{e}\right)=f(p) f\left(p^{e-1}\right)+h(p) f\left(p^{e-1}\right) .
$$

Thus, by Theorem 1 , we have the result.

THEOREM 4. An arithmetical function $f$ is a totient if, and only if, there exists a completely multiplicative function $h$ such that

$$
f(m n)=f(m) \sum_{\substack{d|n \\ \gamma(d)| m}} f(n / d) h(d) .
$$


In this case $f_{v}=h$.

REMARK. Sugunamma ([13], p. 37) has noted that (4) is a necessary condition for totients.

PROOF. Theorem 4 can be proved in a similar way to Theorem 3 .

THEOREM 5. An arithmetical function $f$ is a totient if, and only if, there exist completely multiplicative functions $g$ and $h$ such that

$$
f(m n)=\sum_{d \mid m} \sum_{e \mid n} g(m / d) g(n / e) h^{-1}([d, e]) g^{-1}((d, e)) .
$$

In this case $f_{t}=g$ and $f_{v}=h$. Here $[d, e]$ denotes the least common multiple of $d$ and $e$.

REMARK. Vaidyanathaswamy ([14], p. 645) has noted that (5) is a necessary condition for totients.

PROOF. Formula (5) can be proved by showing that both sides of (5) are multiplicative functions in two variables and by studying prime powers. The converse part follows by taking $n=1$ in (5).

DEFINITION. A divisor $d$ of $n$ is said to be a unitary divisor (or a block divisor) of $n$ if $(d, n / d)=1$. The greatest common unitary divisor of $m$ and $n$ is denoted by $(m, n)_{U}$.

- THEOREM 6. If $f$ is a totient, then

$$
f(m n)=\sum_{d \mid(m, n)} f(m / d) f(n / d) f_{t}(d) f_{v}(d)
$$

whenever $(m, n)_{U}=1$. Conversely if there exists a multiplicative function $F$ such that

$$
f(m n)=\sum_{d \mid(m, n)} f(m / d) f(n / d) F(d)
$$

whenever $(m, n)_{U}=1$, and $f\left(p^{2}\right) \neq f(p)^{2}+F(p), f(p) \neq 0$ for all primes $p$, then $f$ is a totient with $f_{t}(p)=\sqrt{f\left(p^{2}\right)+F(p)}$ for all primes $p$.

REMARK. Identity $\left(6^{\prime}\right)$ is termed as the Busche-Ramanujan identity in the literature. Identity $\left(6^{\prime}\right)$ with the restriction $(m, n)_{U}=1$ is called the restricted Busche-Ramanujan identity. It is well-known that every totient satisfies the restricted Busche-Ramanujan identity, see e.g. [9], p. 53, [14], p. 655. The converse part follows after laborious elementary computations. We do not present the details here. The converse part has been studied in more detail in [6], §3.2.

THEOREM 7. An arithmetical function $f$ is a totient with $f_{t}(p) \neq 0$ for all primes $p$ if, and only if, there exist a completely multiplicative function $g$ with $g(p) \neq 0$ and a complex number $b(p)$ for all primes $p$ such that

$$
f(n)=g(n) \prod_{p \mid n}\left(1-\frac{b(p)}{g(p)}\right) .
$$

In this case $f_{t}=g$ and $f_{v}(p)=b(p)$ for all primes $p$.

REMARK. The classical Dedekind totient $\psi$ is defined as

$$
\psi(n)=n \prod_{p \mid n}\left(1+\frac{1}{p}\right) .
$$

THEOREM 8. If $f$ is a totient, then

$$
f(n) \prod_{p \mid n} f_{t}(p)=f_{t}(n) \prod_{p \mid n} f(p)
$$


Conversely, if for all $n$

$$
f(n) \prod_{p \mid n} g(p)=g(n) \prod_{p \mid n} f(p),
$$

where $g$ is completely multiplicative with $g(p) \neq 0$ for all primes $p$, then $f$ is a totient with $f_{t}=g$.

DEFINITION. We define $\gamma$ to be the multiplicative function such that $\gamma\left(p^{a}\right)=p$ for all primes $p$.

THEOREM 9. If $f$ is a totient, then

$$
f_{t}(n) f(m)=f_{t}(m) f(n)
$$

whenever $\gamma(m)=\gamma(n)$. Conversely, if $f$ is multiplicative and if there exists a completely multiplicative function $g$ such that $g(p) \neq 0$ for all primes $p$ and

$$
g(n) f(m)=g(m) f(n),
$$

whenever $\gamma(m)=\gamma(n)$, then $f$ is a totient with $f_{t}=g$.

REMARK. Formula (9) is well-known for the Euler totient $\phi$ (see e.g. [2], Exercise 2.2).

Theorems 7-9 followfrom the definition of totients and properties of completely multiplicative functions. Details of proofs are left to the reader.

DEFINITION. We say that an arithmetical function $f$ satisfies property $O$ if $f(p)=0$ for a prime $p$ implies $f\left(p^{e}\right)=0$ for all $e>1$.

THEOREM 10. An arithmetical function $f$ is a totient if, and only if, $f$ satisfies property $O$ and there exists a completely multiplicative function $g$ such that

$$
f(m n) f((m, n))=f(m) f(n) g((m, n)) .
$$

In this case $f_{t}=g$.

REMARK. For material relating to the functional equation (10) we refer to [3], [4] and [5].

PROOF. Formula (10) can easily be seen to be valid when $m$ and $n$ are prime powers. Since, in addition, both sides of (10) are multiplicative functions in $m$ and $n$, we have (10). The converse part follows by taking $(m, n)=1$ and $m=p^{e-1}, n=p(e \geq 2)$ in (10) and applying Theorem 1.

COROLLARY 3. If $f$ is an integer-valued totient, then

$$
a|b \Rightarrow f(a)| f(b)
$$

PROOF. Since $a \mid b$, we have $b=a c$. If $c=b$, then $a=1$ and the result is true. Suppose that $c<b$. By $(10)$ we have

$$
f(b) f((a, c))=f(a c) f((a, c))=f(a) f(c) f_{t}((a, c)) .
$$

If $f((a, c))=0$, then we can conclude that $f(b)=0$ and the corollary is valid. Suppose that $f((a, c)) \neq 0$. Then

$$
f(b)=f(a) f(c) f_{t}((a, c)) / f((a, c)) .
$$

Now we can conclude the result inductively. 
REMARK. Formula (10) and the corollary are well-known for the Euler totient (see e.g. [2], Theorem 2.5).

DEFINITION. Let $(m, n)_{k}$ denote the greatest divisor of $n$ which is a $k$ th power divisor of $m$.

THEOREM 11. Let $f$ and $g$ be arithmetical functions and let $f(n) \neq 0$ for all $n$. If $f$ and $g$ are totients with $f_{v}=g_{t}$, then

$$
\frac{(f * g)(n)}{f(n)}=\frac{(f * g)(\delta)}{f(\delta)} \sum_{\substack{d \mid n \\\left(m, d^{k}\right)_{k}=1}} \frac{g^{-1}(d)}{f(d)} \mu(d), \text { where } \delta^{k}=\left(m, n^{k}\right)_{k},
$$

for all $m$ and $n$. Conversely, if there exist completely multiplicative functions $\alpha, \beta$ and $\eta$ such that $\left(\alpha * \beta^{-1}\right)(n)-\mu(n)\left(\beta * \eta^{-1}\right)(n) \neq 0$ for all $n$ and

$$
\frac{\left(\alpha * \beta^{-1}\right)(n)}{f(n)}=\frac{\left(\alpha * \beta^{-1}\right)(\delta)}{f(\delta)} \sum_{\substack{d \mid n \\\left(m, d^{k}\right)_{k}=1}} \frac{\left(\beta * \eta^{-1}\right)(d)}{f(d)} \mu(d)
$$

for all $n$ and for some $m=p^{e}(0 \leq e \leq k)$, then $f$ is a totient with $f_{t}=\alpha$.

PROOF. Formula (11) is a direct consequence of Theorem 9 of [7].

Assume that $\left(11^{\prime}\right)$ holds. We shall firstly prove that $f$ is multiplicative. We proceed by induction on $r s$ to prove that $f(r s)=f(r) f(s)$ whenever $(r, s)=1$. This is valid for $r s=1$. Assume that $f(a b)=f(a) f(b)$ when $(a, b)=1, a b<r s$. We distinguish three cases.

Case 1. Let $\left(m, r^{k} s^{k}\right)_{k}=1$. Then by the inductive assumption,

$$
\begin{aligned}
\frac{\left(\alpha * \beta^{-1}\right)(r s)}{f(r s)}= & \frac{\left(\alpha * \beta^{-1}\right)(r)}{f(r)} \frac{\left(\alpha * \beta^{-1}\right)(s)}{f(s)}+\frac{\left(\beta * \eta^{-1}\right)(r s)}{f(r s)} \mu(r s) \\
& -\frac{\left(\beta * \eta^{-1}\right)(r)}{f(r)} \mu(r) \frac{\left(\beta * \eta^{-1}\right)(s)}{f(s)} \mu(s) .
\end{aligned}
$$

Therefore $f(r s)=f(r) f(s)$.

Case 2. Let $\left(m, r^{k} s^{k}\right)_{k}=r^{k} s^{k}$. As $m$ is a prime power and $(r, s)=1$, we have $r=1$ or $=1$. Thus $f(r s)=f(r) f(s)$.

Case 3. Let $1<\left(m, r^{k} s^{k}\right)_{k}<r^{k} s^{k}$. Then, by the inductive assumption and (11'),

$$
\frac{\left(\alpha * \beta^{-1}\right)(r s)}{f(r s)}=\frac{\left(\alpha * \beta^{-1}\right)(r)}{f(r)} \frac{\left(\alpha * \beta^{-1}\right)(s)}{f(s)} .
$$

Thus $f(r s)=f(r) f(s)$.

Now we have proved that $f$ is multiplicative. Secondly we shall prove that $f\left(p^{a}\right)=$ $\alpha\left(p^{a-1}\right) f(p)$ for all prime powers $p^{a}$. We consider two cases.

Case 1. Let $p^{k} \not$ ' $m$. Taking $n=p$ in $\left(11^{\prime}\right)$ gives $f(p)=\alpha(p)-\eta(p)$. Then taking $n=p^{a}$ in $\left(11^{\prime}\right)$ proves that $f\left(p^{a}\right)=\alpha\left(p^{a-1}\right) f(p)$.

Case 2. Let $p^{k} \mid m$. As $m=p^{e}, 0 \leq e \leq k$, we have $e=k$. Thus taking $n=p^{a}$ in (11') gives $f\left(p^{a}\right)=\alpha\left(p^{a-1}\right) f(p)$. By Theorem 1 we now obtain the result.

THEOREM 12. Let $f$ and $g$ be arithmetical functions such that $(f * g)(n) \neq 0$ for all $n$. If $f$ and $g$ are totients with $f_{v}=g_{t}$, then

$$
\frac{f(n)}{(f * g)(n)}=\frac{f(\delta)}{(f * g)(\delta)} \sum_{\substack{d \mid n \\\left(m, d^{k}\right)_{k}=1}} \frac{g(d)}{(f * g)(d)} \mu(d), \text { where } \delta^{k}=\left(m, n^{k}\right)_{k},
$$


for all $m$ and $n$. Conversely, if if there exist completely multiplicative functions $\alpha, \beta$ and $\eta$ such that $\left(\alpha * \beta^{-1}\right)(n) \neq 0$ for all $n$ and such that

$$
\frac{f(n)}{\left(\alpha * \beta^{-1}\right)(n)}=\frac{f(\delta)}{\left(\alpha * \beta^{-1}\right)(\delta)} \sum_{\substack{d \mid n \\\left(m, d^{k}\right)_{k}=1}} \frac{\left(\eta * \beta^{-1}\right)(d)}{\left(\alpha * \beta^{-1}\right)(d)} \mu(d)
$$

for all $n$ and for $m=p^{e}(0 \leq e \leq k)$, then $f$ is a totient with $f:=c$

Theorem 12 can be proved in a similar way to Theorem 11 . We omit the details.

REMARK. Identities (11) and (12) can be considered as generalized Landau identities. The classical Landau identity [8] is

$$
\sum_{d \mid n} \frac{\mu^{2}(d)}{\phi(d)}=\frac{n}{\phi(n)}
$$

The classical Brauer-Rademacher identity (see e.g. [9], p. 75) is

$$
\phi(n) \sum_{\substack{d \mid n \\(m, d)=1}} \frac{d}{\phi(d)} \mu(n / d)=\mu(n) \sum_{d \mid(m, n)} d \mu(n / d) .
$$

This identity could also be used as a source of characterizations of totients in a similar way to the Landau identity. We do not present details. The Brauer-Rademacher and the Landau type identities have been considered as characterizations of totients in a very general setting in [7].

THEOREM 13. An arithmetical function $f$ is a totient if, and only if, $f$ is multiplicative and if for all primes $p$ there exists complex numbers $a(p)$ and $b(p)$ such that

$$
f_{p}(x)=\frac{1-b(p) x}{1-a(p) x}
$$

In this case $f_{t}(p)=a(p)$ and $f_{v}(p)=b(p)$.

PROOF. If $f$ is a totient, then

$$
f_{p}(x)=\left(f_{t} * f_{v}^{-1}\right)_{p}(x)=\frac{\left(f_{f}\right) \cdot(x)}{\left(f_{v}\right)_{p}(x)}=\frac{1-f_{v}(p) x}{1-f_{t}(p) x} .
$$

Conversely, let $g$ and $h$ be completely multiplicative functions defined by $g(p)=a(p)$ and $h(p)=b(p)$. Then $f_{p}(x)=\left(g * h^{-1}\right)_{p}(x)$ at all primes $p$; hence $f=g * h^{-1}$. This completes the proof.

Theorems 1-12 could easily be generalized to the setting of Narkiewicz's regular convolution (see [9], Chapter 4). We do not present the details here.

\section{AN EXAMPLE}

The arithmetical function $\theta(n)$ is defined as the number of ordered pairs $\langle a, b\rangle$ such that $(a, b)=1$ and $a b=n$. It is easy to see that $\theta(n)=2^{\omega(n)}$, where $\omega(n)$ is the number of distinct prime factors of $n$ with $\omega(1)=0$. Also $\theta(n)$ is the number of squarefree divisors of $n$. This suggests the standard generalization $\theta_{k}$ of $\theta$. In fact, $\theta_{k}(n)$ is the number of $k$-free divisors of $n$ (see e.g. $\left[9\right.$, p. 36, 37]). In particular, $\theta_{2}=\theta$. The definition of $\theta(n)$ however suggests we look upon another generalization of $\theta(n)$. We define $\theta_{(k)}(n)$ to be the number of ordered $k$-tuples $\left\langle a_{1}, a_{2}, \ldots, a_{k}\right\rangle$ such that $a_{i}$ 's are relatively prime and $a_{1} a_{2} \cdots a_{k}=n$. It is easy to see that

$$
\theta_{(k)}(n)=k^{\omega(n)}
$$


The generating series of $\theta_{(k)}$ is

$$
\left(\theta_{(k)}\right)_{p}(x)=\frac{1-(1-k) x}{1-x}
$$

at all primes $p$. If we define the function $\lambda_{(1-k)}$ by $\lambda_{(1-k)}(n)=(1-k)^{\Omega(n)}$, where $\Omega(n)$ is the total number of prime divisors of $n$ each counted according to its multiplicity, then by Theorem 13

$$
\theta_{(k)}(n)=\left(e * \mu \lambda_{(1-k)}\right)(n)
$$

where $e \equiv 1$. The function $\lambda_{(1-k)}$ is a generalized Liouville function with $\lambda_{(-1)}=\lambda$, where $\lambda$ is the classical Liouville function. Thus $\theta=\theta_{(2)}=e * \mu \lambda=e * \lambda^{-1}=e * \mu^{2}$. Further, $\lambda_{(1-k)}$ is clearly completely multiplicative; hence $\theta_{(k)}$ possesses the structure of a totient.

Application of formulas (1)-(12) to $\theta_{(k)}$ yields the following identities.

$$
\begin{aligned}
& \theta_{(k)}\left(p^{e}\right)=\theta_{(k)}(p) \text { for all } e \geq 1, \\
& \theta_{(k)}(n)=\sum_{x(\bmod n)} \sum_{d \mid(x, n)}\left(\mu \lambda_{(1-k)}\right)(d)(d / n), \\
& \theta_{(k)}(m) \theta_{(k)}(n)=\sum_{d \mid(m, n)} \theta_{(k)}(m n / d)\left(\mu \lambda_{(1-k)}\right)(d), \\
& \theta_{(k)}(m n)=\theta_{(k)}(m) \sum_{\substack{d|n \\
\gamma(d)| m}} \theta_{(k)}(n / d) \lambda_{(1-k)}(d), \\
& \theta_{(k)}(m n)=\sum_{d \mid m} \sum_{e \mid n} \mu([d, e]) \mu((d, e)) \lambda_{(1-k)}([d, e]) \\
& =\sum_{d \mid m} \sum_{e \mid n} \mu(d) \mu(e) \lambda_{(1-k)}([d, e]), \\
& \theta_{(k)}(m n)=\sum_{d \mid(m, n)} \theta_{(k)}(m / d) \theta_{(k)}(n / d) \lambda_{(1-k)}(d), \quad \text { whenever }(m, n)_{U}=1, \\
& \theta_{(k)}(n)=\prod_{p \mid n} k \\
& \theta_{(k)}(n)=\prod_{p \mid n} \theta_{(k)}(p), \\
& \theta_{(k)}(m)=\theta_{(k)}(n), \quad \text { whenever } \gamma(m)=\gamma(n), \\
& \theta_{(k)}(m n) \theta_{(k)}((m, n))=\theta_{(k)}(m) \theta_{(k)}(n), \\
& \frac{\left(e * h^{-1}\right)(n)}{\theta_{(k)}(n)}=\frac{\left(e * h^{-1}\right)(\delta)}{\theta_{(k)}(\delta)} \sum_{\substack{d \mid n \\
\left(m, d^{k}\right)_{k}=1}} \frac{\left(h *\left(\mu \lambda_{(1-k)}\right)\right)(d)}{\theta_{(k)}(d)} \mu(d), \delta^{k}=\left(m, n^{k}\right)_{k}, \\
& \frac{\theta_{(k)}(n)}{\left(e * h^{-1}\right)(n)}=\frac{\theta_{(k)}(\delta)}{\left(e * h^{-1}\right)(\delta)} \sum_{\substack{d \mid n \\
\left(m, d^{k}\right)_{k}=1}} \frac{\left(\lambda_{(1-k)} * h^{-1}\right)(d)}{\left(e * h^{-1}\right)(d)} \mu(d), \delta^{k}=\left(m, n^{k}\right)_{k}
\end{aligned}
$$

for any completely multiplicative function $h$ with $\left(e * h^{-1}\right)(n) \neq 0$ for all $n$. If $h=e_{0}$, the last two equations reduce to

$$
\begin{aligned}
& \frac{1}{\theta_{(k)}(n)}=\frac{1}{\theta_{(k)}(\delta)} \sum_{\substack{d \mid n \\
\left(m, d^{k}\right)_{k}=1}} \frac{\left(\mu \lambda_{(1-k)}\right)(d)}{\theta_{(k)}(d)} \mu(d), \delta^{k}=\left(m, n^{k}\right)_{k}, \\
& \theta_{(k)}(n)=\theta_{(k)}(\delta) \sum_{\substack{d \mid n \\
\left(m, d^{k}\right)_{k}=1}}\left(\mu \lambda_{(1-k)}\right)(d), \delta^{k}=\left(m, n^{k}\right)_{k} .
\end{aligned}
$$


Some of these identities are trivial. Note that the generating series (13) of $\theta_{(k)}$ is given in (14). Formulas (1)-(13) could also be applied to other concrete totients to obtain identities for these totients. We leave the details to the reader.

Note that $\theta_{(k)}$ could also be presented in terms of the unitary convolution. The unitary convolution $f \oplus g$ of two arithmetical functions $f$ and $g$ is defined by

$$
(f \oplus g)(n)=\sum_{d \| n} f(d) g(n / d),
$$

where $d \| n$ means that $d$ is a unitary divisor of $n$, that is, a divisor $d$ of $n$ with $(d, n / d)=1$. It is easy to see that

$$
\theta_{(k)}=e \oplus e \oplus \cdots \oplus e \quad(e, k \text { times }) .
$$

In particular, $\theta=\theta_{(2)}=e \oplus e$.

\section{REFERENCES}

1. APOSTOL, T. M. Some properties of completely multiplicative arithmetical functions, Amer. Math. Monthly $\underline{78}$ (1971), 266-271.

2. APOSTOL, T. M. Introduction to Analytic Number Theory, Springer-Verlag, New York, 1976.

3. APOSTOL, T. M. and ZUCKERMAN, H. S. On the functional equation $F(m n) F((m, n))=$ $F(m) F(n) f((m, n))$, Pacific J. Math. 14 (1964), 377-384.

4. CHIDAMBARASWAMY, J. On the functional equation $F(m n) F((m, n))=F(m) F(n)$ $f((m, n))$, Portugal. Math. $\underline{26}$ (1967), 101-107.

5. COMMENT, P. Sur l'equation fonctionelle $F(n m) F((n, m))=F(n) F(m) f((n, m))$, Bull. Res. Counc. Israel, Section F, $\underline{7}$ (1957), 14-20.

6. HAUKKANEN, P. Classical arithmetical identities involving a generalization of Ramanujan's sum, Ann. Acad. Sci. Fenn. Ser. A. I. Math. Diss. $\underline{68}$ (1988), 1-69.

7. HAUKKANEN, P. and McCARTHY, P. J. Arithmetical identities of the BrauerRademacher type, Aeq. Math. $\underline{39}$ (1990), 40-54.

8. LANDAU, E. Über die zahlentheoretische Funktion $\phi$ und ihre Beziehung zum Goldbachschen Satz, Göttinger Nachrichten (1900), 177-186.

9. McCARTHY, P. J. Introduction to Arithmetical Functions, Springer-Verlag, New York, 1986.

10. SIVARAMAKRISHNAN, R. Contributions to the study of multiplicative arithmetic functions, Ph. D. Thesis, University of Kerala, 1971.

11. SIVARAMAKRISHNAN, R. Classical Theory of Arithmetic Functıons, Marcel Dekker, Inc., New York, 1989.

12. SIVARAMAKRISHNAN, R. The many facets of Euler's totient. II: Generalizations and analogues, Nieuw Arch. Wiskd., IV. Ser. 8, No. 2 (1990), 169-187.

13. SUGUNAMMA, M. Contributions to the study of general arithmetic functions, $\mathrm{Ph}$. D. Thesis, Sri Venkateswara University, Tirupati, 1965.

14. VAIDYANATHASWAMY, R. The theory of multiplicative arithmetic functions, Trans. Amer. Math. Soc. $\underline{33}$ (1931), 579-662.

15. WALL, C. R. and HSU, T. S. A characterization of totients, Notıces Amer. Math. Soc. $\underline{19}(1972), 567$. 


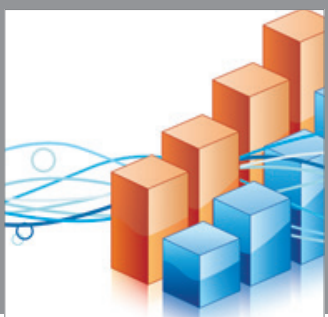

Advances in

Operations Research

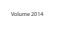

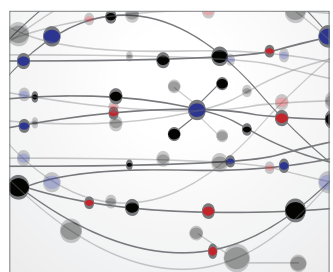

\section{The Scientific} World Journal
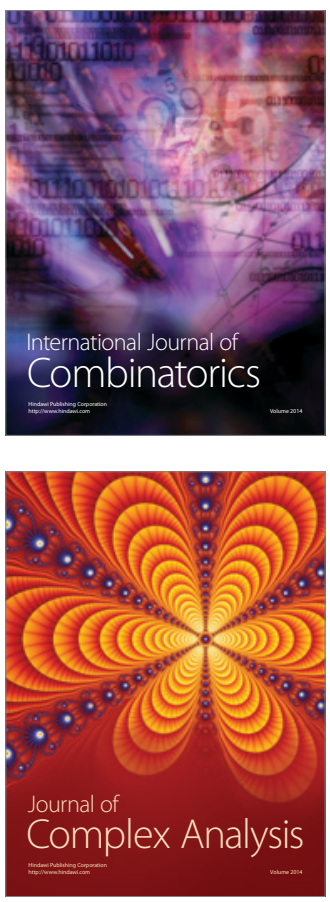

International Journal of

Mathematics and

Mathematical

Sciences
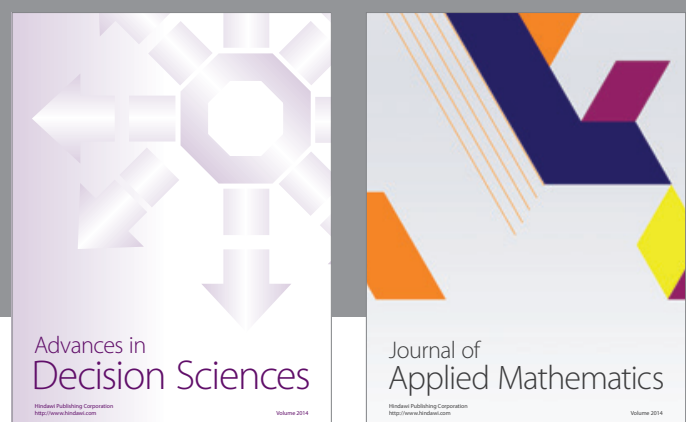

Journal of

Applied Mathematics
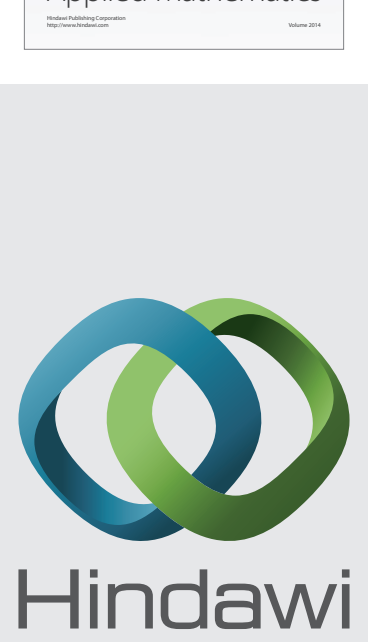

Submit your manuscripts at http://www.hindawi.com
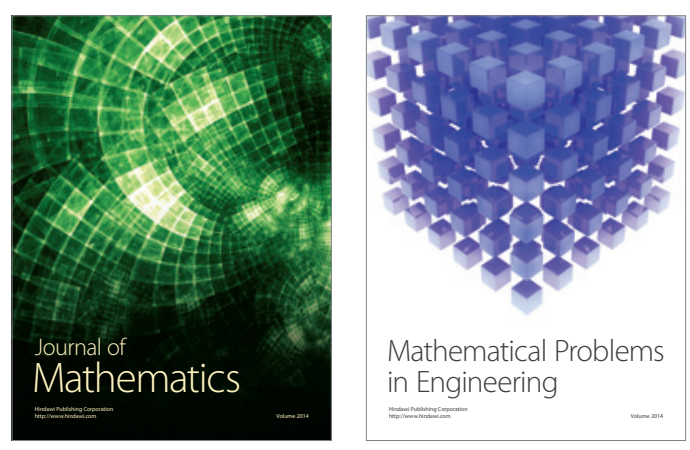

Mathematical Problems in Engineering
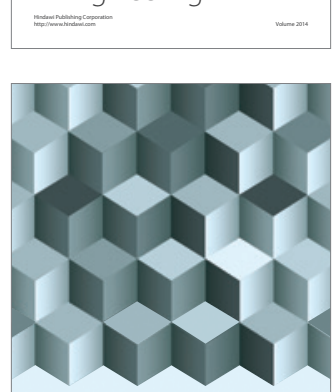

Journal of

Function Spaces
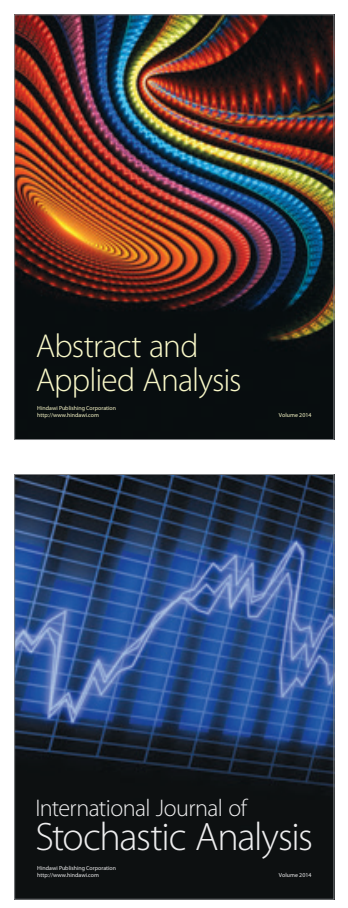

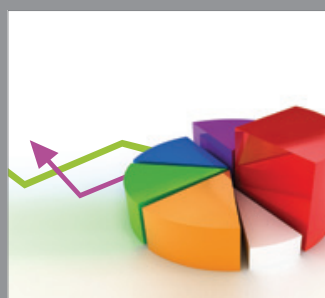

ournal of

Probability and Statistics

Promensencen
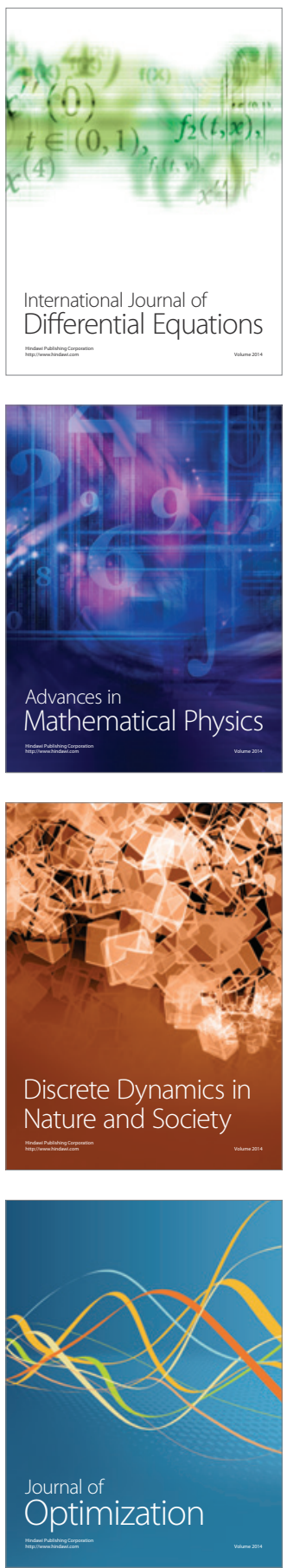